\title{
BMJ Open Effectiveness of Xin Jia Xuan Bai Cheng Qi Decoction in treating acute exacerbation of chronic obstructive pulmonary disease: study protocol for a multicentre, randomised, controlled trial
}

\author{
Jin Jin, ${ }^{1}$ Hongchun Zhang, ${ }^{2}$ Demin Li, ${ }^{3}$ Yue Jing, ${ }^{4}$ Zengtao Sun, ${ }^{5}$ Jihong Feng, ${ }^{6}$ \\ Hong Zhang, ${ }^{7}$ Yan Zhang, ${ }^{7}$ Tianhong Cui, ${ }^{8}$ Xiang Lei, ${ }^{8}$ Jing Zhang, ${ }^{9}$ Qijian Cheng, ${ }^{10}$ \\ Erran $\mathrm{Li}^{11}$
}

To cite: Jin J, Zhang H, Li D, et al. Effectiveness of Xin Jia Xuan Bai Cheng Qi Decoction in treating acute exacerbation of chronic obstructive pulmonary disease: study protocol for a multicentre, randomised controlled trial. BMJ Open 2019;9:e030249. doi:10.1136/ bmjopen-2019-030249

- Prepublication history and additional material for this paper are available online. To view these files, please visit the journal online (http://dx.doi org/10.1136/bmjopen-2019030249).

Received 11 March 2019 Revised 17 September 2019 Accepted 23 September 2019

Check for updates

(c) Author(s) (or their employer(s)) 2019. Re-use permitted under CC BY-NC. No commercial re-use. See rights and permissions. Published by BMJ.

For numbered affiliations see end of article.

Correspondence to Professor Hongchun Zhang; 13701226664@139.com

\section{ABSTRACT}

Introduction Acute exacerbation of chronic obstructive pulmonary disease (AECOPD) brings a serious impact on patients' quality of life, and has extremely high morbidity and mortality worldwide. Although there are many therapies being developed to alleviate symptoms and reduce mortality, a few studies have supported which treatment method is the best. Traditional Chinese medicine (TCM) has shown good potential in the prevention and treatment of $A E C O P D$, especially in terms of supplementation and reduction of dosage and adverse effect of Western medicine. The purpose of this study is to compare the effectiveness of combination of TCM and Western medicine with conventional therapy alone for AECOPD, and to ensure whether the combined therapy may reduce the use of systemic glucocorticoid in AECOPD without influencing efficacy.

Methods and analysis A multicentre, randomised, double-blind, placebo-controlled study was conducted to enrol a total of 360 eligible patients who will be randomised into integrated Chinese and Western medicine group A, B and Western standard Medicine group C. After 5 days of intervention and 1 month of follow-up, the efficacy and safety of Xin Jia Xuan Bai Cheng Qi Decoction in patients with AECOPD will be observed. The results of evaluation indicators include: clinical symptoms, biochemical indicators such as blood gas analysis, inflammatory markers, hospitalisation time, TCM syndrome evaluation, biological indicators such as airway, intestinal flora sequencing.

Ethics and dissemination This trail has been approved by the Ethics Committee of China-Japan Friendship Hospital. The results will be disseminated in international peer-reviewed journals and be presented in academic conferences. The results will also be disseminated to patients by telephone, inquiring on patient's poststudy health status during the follow-up.

Trial registration number ChiCTR1800016915

\section{INTRODUCTION}

Chronic obstructive pulmonary disease (COPD) is a slowly progressive disease

\section{Strengths and limitations of this study}

A randomised, double-blind, placebo-controlled, clinical, critical trial.

- A multicentre trial be carried out in four comprehensive third-grade first-class hospitals across China, which improves the external validity and representativeness of the sample and reduces the risk of selection bias.

- Because clinical symptoms are often accompanied by a certain degree of subjectivity, we combine the core symptoms (cough, phlegm, defecation and dyspnoea) score and traditional Chinese medicine syndrome score to ensure scientific objectivity.

- As the main phase of the programme will be performed during the hospital stay, practitioners will be able to immediately identify and manage adverse reactions to guarantee the safety of participants. Practitioners will maintain good communication with the participants by phone after discharge.

- The 5-day treatment period and the 1-month followup period may be a bit short.

characterised by airflow obstruction (forced expiratory volume in $1 \mathrm{~s} \quad\left(\mathrm{FEV}_{1}\right)<80 \%$ predicted and $\mathrm{FEV}_{1} /$ forced vital capacity ratio $<70 \%$ ) that is not reversible. COPD is currently the fourth-leading cause of death in the world but is projected to be the thirdleading cause of death by $2020 .{ }^{1}$ In China, the prevalence of COPD in people aged 40 years or older is $13.7 \%,{ }^{2}$ more than 1 million people die and more than 5 million people be disabled due to COPD each year.

Acute exacerbation of COPD (AECOPD) ${ }^{3}$ is defined as acute worsening of respiratory symptoms(typically dyspnoea, cough, increased sputum and/or purulent sputum) in patients with COPD, exceeding normal day-to-day variations that may 
require a change in medication even hospitalisation. Acute exacerbation is an important factor in the death of patients with COPD ${ }^{4}$ and is also the main expenditure portion of medical expenses for patients with COPD.

Traditional Chinese medicine (TCM), with characteristic theory of syndrome differentiation and overall conditioning, implies its potential advantages in the treatment of COPD. TCM syndrome studies find that in addition to symptoms such as cough, phlegm and wheezing, patients in acute exacerbation period are often accompanied by constipation, abdominal distension and yellow greasy tongue coating. Based on TCM characteristic theory 'the Lung and the Large Intestine Are Interior-Exteriorl', physicians use the method of Tongfu Xiere to patients with AECOPD and usually achieve good results in clinical practice. Xin Jia Xuan Bai Cheng Qi Decoction (XJXBCQ), consists of Armeniacae Semen Amarum, Gypsum Fibrosum, Trichosanthis Fructus, Rhei Radix Et Rhizoma, Scutellariae Radix, Perillae Fructus, Glycyrrhizae Radix Et Rhizoma Praeparata Cum Melle, Fagopyri Dibotryis Rhizoma and Asteris Radix Et Rhizoma, is evolved from the TCM classical prescription XJXBCQ. ${ }^{5}$ AECOPD is often treated with antibiotics and glucocorticoid with the risk of excessive dosage and unnecessary adverse effect, ${ }^{6}$ where TCM can play a complementary and alternative role. TCM has been widely used in patients with AECOPD attached to the heat-phlegm and sthenic-fu syndrome, especially in combination with Western medicine, not only can reduce the use of antibiotics, glucocorticoid, but also decrease the adverse effect of modern routine medicine. Despite this, there is not enough evidence to show the efficacy of XJXBCQ on patients of AECOPD. Hence, a more rigorously designed large-scale, multicentre, randomised trial is needed to assess the effectiveness of XJXBCQ on AECOPD. In conclusion, our aim is to conduct a multicentre, randomised, doubleblind, placebo-controlled trial to evaluate the efficacy and safety of XJXBCQ on AECOPD. The results of this trial will provide evidence that XJXBCQ is an effective prescription for AECOPD. In view of the difficulty in assessing the quality of the decoction, we plan to use the XJXBCQ granules in the trial. The main purpose of this study is to evaluate the clinical symptoms, signs, blood gas analysis, serum inflammatory factors (interleukin-6 (IL-6), ${ }^{7}$ tumour necrosis factor- $\alpha(\mathrm{TNF}-\alpha)$, C reactive protein (CRP) ${ }^{8}$ procalcitonin (PCT)), airway and intestinal microbes ${ }^{9}$ of AECOPD hospitalised patients with XJXBCQ combined with Western medicine. The secondary objective was to observe the effect of XJXBCQ combined with Western medicine on reducing the use of glucocorticoid, ${ }^{10}$ the mortality, the hospitalised time, the requirement for invasive mechanical ventilation and the readmission rate of acute exacerbation within 30 days after discharge.

\section{METHODS}

\section{Study design}

This is a multicentre, randomised, double-blind, placebocontrolled clinical trial that enrolled a total of 360 patients that will be randomly assigned to one of three groups: integrated Chinese and Western medicine group A, integrated Chinese and Western medicine group B and Western standard medicine group C. After 5 days of intervention, the effectiveness and safety of XJXBCQ in participants will be evaluated by comparing the various indicators of the three groups, including clinical symptoms, activity, biochemical indicators and biological indicators.

As the leading unit of the research, China-Japan Friendship Hospital is responsible for training the standard operating procedures (SOPs) of researchers and supervising the progress of all clinical sites. Other participating units include: Zhongshan Hospital affiliated to Fudan University, the First Affiliated Hospital of China Medical University and Ruijin Hospital affiliated to School of Medicine, Shanghai Jiao Tong University. Recruitment allocation: 180 cases will be from China-Japan Friendship Hospital, while the remaining three recruit 60 cases, respectively. The flow chart of the trial is shown in figure 1 . This study protocol is registered with the China Clinical Trial Registry (http://www.chictr.org.cn/showproj.aspx?proj= 28338). The Standard Protocol Items: Recommendation for Interventional Trials 2013 checklist is shown in online additional file 1 .

\section{Participants}

Patients with AECOPD attached to the heat-phlegm and sthenic-fu syndrome will be enrolled. Heat-phlegm and sthenic-fu syndrome is a typical syndrome type of TCMbased TCM characteristic theory 'the Lung and the Large Intestine Are Interior-Exteriorl'. Referred to 'Retention of Heat-Phlegm in the Lung' syndrome in TCM Diagnosis and Treatment Guidelines for COPD (2011), Heat-phlegm and sthenic-fu syndrome is defined as: primary symptoms: cough, wheezing, chest distress, yellow and white sticky sputum, abdominal distension, constipation, red tongue, yellow and greasy fur, slippery or rapid pulse; secondary symptoms: chest pain, facial blushing, thirst with desire to cold drinks, yellow urine, thick fur. Diagnosis: (1) cough or shortness of breath, (2) yellow and white sticky sputum with difficult expectoration, (3) abdominal distension or constipation, (4) facial blushing, (5) thirst with desire to cold drinks, (6) yellow urine, (7) red tongue, yellow and greasy fur, slippery or rapid pulse. The diagnosis should meet $(1,2)$ and $(3)$, and two of $(4,5,6)$ or $(7)$. The first date of participant enrolment was 7 January 2019.

\section{Inclusion criteria}

Patients must meet all of the following criteria: (1) Meet AECOPD diagnostic criteria; (2) AECOPD severity clinical grade $\mathrm{I}-\mathrm{II}^{11}$; (3) comply with indications for antibiotic treatment recommended by Chinese Expert Consensus on diagnosis and treatment of AECOPD (Updated 


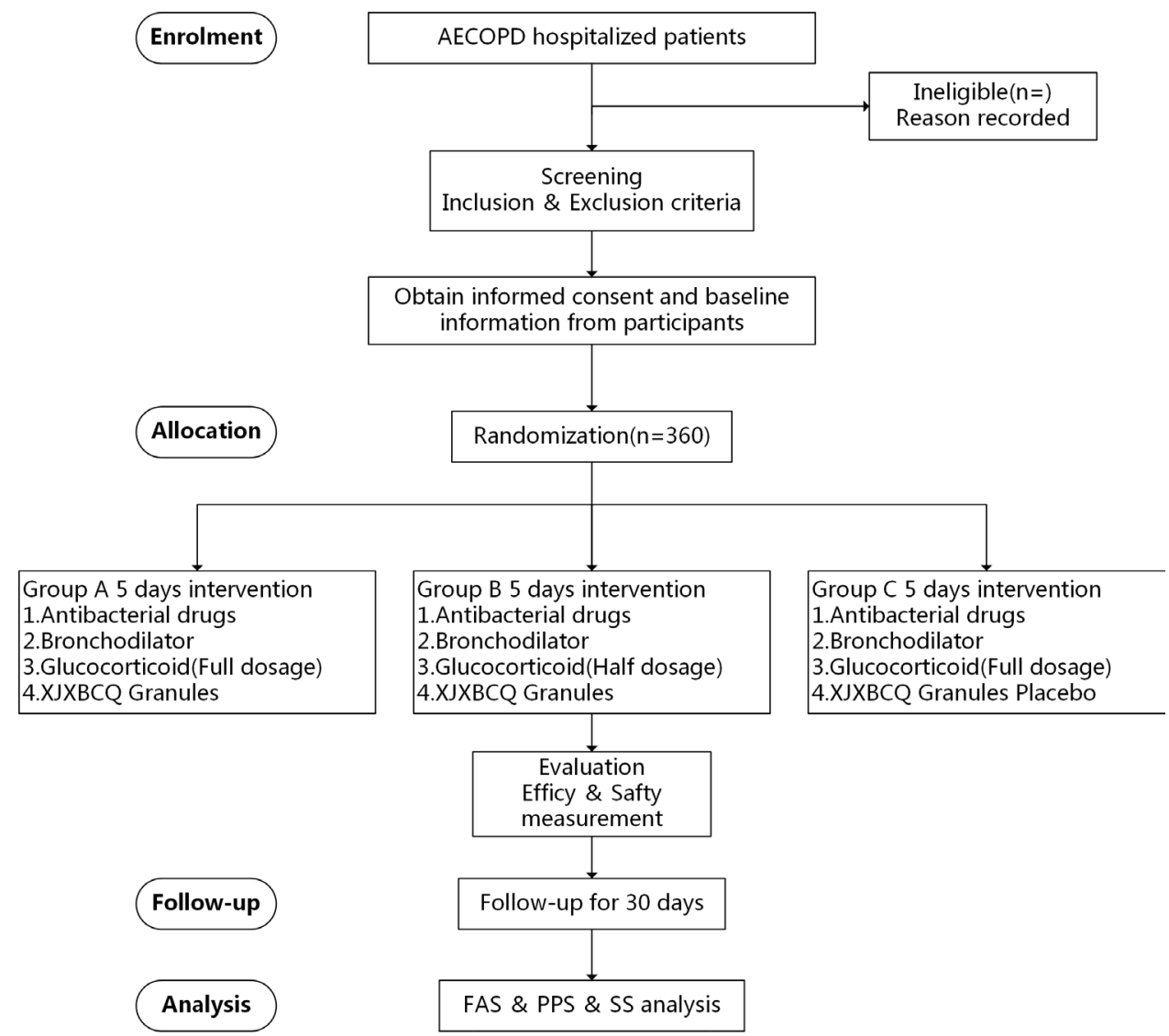

Figure 1 Study flow chart. AECOPD, acute exacerbation of chronic obstructive pulmonary disease; FAS, full analysis set; PPS, per protocol set; SS, safety analysis set; XJXBCQ, Xin Jia Xuan Bai Cheng Qi Decoction.

2017); (4) comply with the criteria for the heat-phlegm and sthenic-fu syndrome of TCM; (5) age 40-80 years old, gender is not limited and (6) provision of signed, informed consent.

\section{Exclusion criteria}

Patients who meet one or more of the exclusion criteria listed below will not be allowed: (1) patients who with asthma, pneumonia, bronchiectasis, cystic fibrosis, pulmonary tuberculosis, lung cancer or any other airflow-limited disease with known causes and characteristic pathology; (2) patients with coronary heart disease, hypertensive heart disease or heart valve disease; (3) those need invasive mechanical ventilation; (4) clinically confirmed or highly suspected pulmonary embolism; (5) combine with diseases of severe cardiovascular, cerebrovascular, hepatorenal and haematopoietic or primary endocrine system $^{12} ;(6)$ those with intestinal obstruction requiring surgical intervention; (7) pregnant or lactation period; (8) mentally handicapped; (9) Alanine aminotransferase (ALT), aspartate aminotransferase (AST) $>1.5$ times the upper limit of normal reference or serum creatinine (Scr) >the upper limit of normal reference; (10) need to combine immunosuppressant; (11) taking oral or intravenous antibiotics before screening for more than 3 days in last 3 months; (12) known to be allergic to the basic therapeutic drugs or other excipients prescribed through the research; (13) known to be allergic to Chinese herbal medicinal ingredient prescription; (14) those who have participated in or are participating in other clinical trials in last 3 months and (15) those who be considered inappropriate to participate in this clinical trial by the investigator.

\section{Sample size}

According to our pre-experiment, comparing symptom scores of patients before and after treatment, the average score was $10.67 \pm 2.61$ on admission, $4.33 \pm 1.97$ on the 6 th day of admission, and the difference value was $6.33 \pm 3.73$. Excellent efficiency test will be conducted between integrated Chinese and Western medicine Group A and Western standard medicine group C, meanwhile noninferiority test will be conducted between integrated Chinese and Western medicine group B and Western standard medicine group C. Cut-off point is defined as 1, so expected difference of excellent efficiency test should be more than $7.33(6.33+1)$, and which of non-inferiority test should be not less than $5.33(6.33-1)$. When the power $=0.8$, each of the three groups required 100 effective cases. Considering a $20 \%$ shedding rate, a total of 360 cases were needed in the three groups, with 120 cases in each group.

\section{Randomisation}

A block randomisation method will be employed. We will select the appropriate length of the segment and use the 
SAS statistical software to generate a random sequence of 360 subjects (group A, group B, group C) according to the ratio of $1: 1: 1$, listing the serial number as $001-360$. The treatment allocation is that each centre will be assigned a consecutive numbered medication on the basis of the random sequence. An independent clinical statistician will record the method, process, result of entire produce and keep the random sequence which be saved as a file in a sealed envelope so as to be checked if necessary. In case there is a clinical emergency event, the individual's randomised code and group assignment can be identified as quickly as possible through the emergency envelope. Once any envelope has been opened, whether intentional or not, it should be carefully recorded on the case report form (CRF) and the patient will be withdrawn from the study.

\section{Interventions}

Program description

Volunteers who meet the inclusion criteria will receive an information form and be required to provide written consent to participate in the trial. They will then undergo a physical screening test to determine if there are other comorbidities that may impact the trial. After successful screening, they will participate in the trial, get trialspecific identification numbers and be assigned to a group according to random sequence. A baseline measurement for each participant is then performed, including clinical symptoms scores and TCM syndrome scores, blood gas analysis $\left(\mathrm{PH}\right.$, arterial oxygen tension or pressure, $\mathrm{PaO}_{2}$, alveolar carbon dioxide tension or pressure, $\mathrm{PaCO}_{2}$ ), serum inflammatory markers (PCT, CRP, IL-6, TNF- $\alpha$ ), induced sputum, stool sample and so on. The research programme flow is shown in table 1 . All outcome measurements will be taken by medical workers who are familiar

Table 1 Data collected from baseline to follow-up visits

\begin{tabular}{|c|c|c|c|c|c|c|c|c|c|c|}
\hline & \multicolumn{10}{|c|}{ Study period } \\
\hline & \multirow{2}{*}{$\begin{array}{l}\text { Enrolment } \\
\text { D-1 }\end{array}$} & \multirow{2}{*}{$\begin{array}{l}\text { Allocation } \\
\text { DO }\end{array}$} & \multicolumn{5}{|c|}{ Intervention } & \multicolumn{3}{|c|}{ Postintervention } \\
\hline & & & D1 & D2 & D3 & D4 & D5 & D6 & Discharge & $\begin{array}{l}30 \text { days after } \\
\text { discharge }\end{array}$ \\
\hline \multicolumn{11}{|l|}{ Enrolment } \\
\hline Consent form & $\sqrt{ }$ & & & & & & & & & \\
\hline Basic information & $\sqrt{ }$ & & & & & & & & & \\
\hline Inclusion and exclusion criteria & $\sqrt{ }$ & & & & & & & & & \\
\hline Allocation & & $\sqrt{ }$ & & & & & & & & \\
\hline \multicolumn{11}{|l|}{ Intervention: } \\
\hline XJXBCQ+budesonide (All)+WST & & & $\sqrt{ }$ & $\sqrt{ }$ & $\sqrt{ }$ & $\sqrt{ }$ & $\sqrt{ }$ & & & \\
\hline XJXBCQ+budesonide (half) +WST & & & $\sqrt{ }$ & $\sqrt{ }$ & $\sqrt{ }$ & $\sqrt{ }$ & $\sqrt{ }$ & & & \\
\hline $\begin{array}{l}\text { XJXBCQ placebo +budesonide } \\
\text { (All)+WST }\end{array}$ & & & $\sqrt{ }$ & $\sqrt{ }$ & $\sqrt{ }$ & $\sqrt{ }$ & $\sqrt{ }$ & & & \\
\hline \multicolumn{11}{|l|}{ Outcome measure } \\
\hline Clinical symptoms score & $\sqrt{ }$ & & $\sqrt{ }$ & $\sqrt{ }$ & $\sqrt{ }$ & $\sqrt{ }$ & $\sqrt{ }$ & $\sqrt{ }$ & $\sqrt{ }$ & \\
\hline TCM syndrome score & $\sqrt{ }$ & & $\sqrt{ }$ & $\sqrt{ }$ & $\sqrt{ }$ & $\sqrt{ }$ & $\sqrt{ }$ & $\sqrt{ }$ & $\sqrt{ }$ & \\
\hline $\begin{array}{l}\text { Blood gas analysis }\left(\mathrm{PH}, \mathrm{PaO}_{2} \text {, }\right. \\
\left.\mathrm{PaCO}_{2}\right)\end{array}$ & $\sqrt{ }$ & & & & & & & $\sqrt{ }$ & & \\
\hline $\begin{array}{l}\text { Serum inflammatory markers (PCT, } \\
\text { CRP, IL-6, TNF- } \alpha \text { ) }\end{array}$ & $\sqrt{ }$ & & & & $\sqrt{ }$ & & & $\sqrt{ }$ & & \\
\hline Induced sputum and stool sample & $\sqrt{ }$ & & & & & & & $\sqrt{ }$ & & \\
\hline \multicolumn{11}{|l|}{ Safety assessments: } \\
\hline Adverse events recorded & $\sqrt{ }$ & & $\sqrt{ }$ & $\sqrt{ }$ & $\sqrt{ }$ & $\sqrt{ }$ & $\sqrt{ }$ & $\sqrt{ }$ & $\sqrt{ }$ & $\sqrt{ }$ \\
\hline Physical examination & $\sqrt{ }$ & & $\sqrt{ }$ & $\sqrt{ }$ & $\sqrt{ }$ & $\sqrt{ }$ & $\sqrt{ }$ & $\sqrt{ }$ & $\sqrt{ }$ & \\
\hline Blood and urine routine & $\sqrt{ }$ & & & & & & & $\sqrt{ }$ & & \\
\hline $\begin{array}{l}\text { Liver function (AST, ALT, Tbil, ALP, } \\
\text { GGT ) }\end{array}$ & $\sqrt{ }$ & & & & & & & $\sqrt{ }$ & & \\
\hline Kidney function (Scr, BUN, eGFR ) & $\sqrt{ }$ & & & & & & & $\sqrt{ }$ & & \\
\hline ECG & $\sqrt{ }$ & & & & & & & $\sqrt{ }$ & & \\
\hline
\end{tabular}


with the management of these assessments and will be unaware of the participants' group assignments.

All participants will receive standard Western medicine treatment follow the Global Initiative for Chronic Obstructive Pulmonary Disease 2018, the Chinese Medical Association Guidelines for the Diagnosis and Treatment of COPD (Revised 2013) and the Chinese Expert Consensus on diagnosis and treatment of AECOPD (Updated 2017), including:

General treatment: controlled oxygen therapy, venturi mask oxygen, NIV if requiring non-invasive ventilation;

Antibiotic: levofloxacin (LVFX) and sodium chloride injection, $0.5 \mathrm{~g}$, intravenous.gtt, once a day;

Bronchodilator $^{13}$ : compound ipratropium bromide solution for inhalation (combivent), $500 \mathrm{ug}$, inhal, three times a day.

Beside the above, group A will be given budesonide suspension for inhalation (PULMICORT RESPULES, $1 \mathrm{mg}$ ) (two at a time, two times a day) and XJXBCQ granules (two bags at a time, three times a day). Group B will be given budesonide suspension for Inhalation (PULMICORT RESPULES, $1 \mathrm{mg}$ ) (one at a time, two times a day) and XJXBCQ granules (two bags at a time, three times a day); group $\mathrm{C}$ will be given budesonide suspension for inhalation (PULMICORT RESPULES, $1 \mathrm{mg}$ ) (two at a time, two times a day) and XJXBCQ granules Placebo (two bags at a time, three times a day). The entire trial will go through 5 days.

\section{XJXBCQ granules}

XJXBCQ granules is a compound preparation of Chinese herbal medicine. The main components are shown in table 2.

XJXBCQ granules (2.5 g/bag, batch number: 180605) are produced and packaged by Anhui Jiren Pharmaceutical that have China Pharmaceutical Production License (Number: Wan 20160083). The results of drug quality testing are consistent with the quality standards from Pharmacopoeia of the People's Republic of China 2015.

\begin{tabular}{llc}
\hline $\begin{array}{l}\text { Table } 2 \\
\text { Decoction }\end{array}$ & Main components of Xin Jia Xuan Bai Cheng Qi \\
\hline Chinese name & Latin name & Amount (g) \\
\hline Ku Xing Ren & $\begin{array}{l}\text { Armeniacae Semen } \\
\text { Amarum }\end{array}$ & $6 \mathrm{~g}$ \\
Sheng Shi Gao & Gypsum Fibrosum & $15 \mathrm{~g}$ \\
Gua Lou & Trichosanthis Fructus & $9 \mathrm{~g}$ \\
Da Huang & Rhei Radix Et Rhizoma & $6 \mathrm{~g}$ \\
Huang Qin & Scutellariae Radix & $9 \mathrm{~g}$ \\
Zi Su Zi & Perillae Fructus & $9 \mathrm{~g}$ \\
Zhi Gan Cao & Glycyrrhizae Radix Et & $6 \mathrm{~g}$ \\
& $\begin{array}{l}\text { Rhizoma Praeparata Cum } \\
\text { Jin Qiao Mai }\end{array}$ & Felle \\
Zi Wanopyri Dibotryis Rhizoma & Asteris Radix Et Rhizoma & $9 \mathrm{~g}$ \\
\hline
\end{tabular}

XJXBCQ granules will be administered orally, two bags at a time, three times a day for 5 days. All herbs were tested to the same standard.

\section{Placebo}

The placebo consists of starch without any active ingredient is produced by the same manufacturer as XJXBCQ granules. It is a dextrin that matches as much as possible the appearance and taste of XJXBCQ granules. The drug instructions for XJXBCQ granules and placebo are completely consistent.

\section{Comorbidities and exacerbations regulation}

Participants are allowed to remain their originally basic treatment (such as medicine for hypertension or diabetes) taken before recruited. If one's condition deteriorates during the study and need to stay in the Intensive Care Unit (ICU) or perform invasive mechanical ventilation, we will immediately deal with it. Those who need to adjust the antibiotic due to they can't tolerate LVFX or whose condition have not been alleviated but even aggravated after 3 days of treatment, the antibacterial therapy will be adjusted according to the regulations in the Chinese Expert Consensus for the Treatment of AECOPD (Updated 2017). The reasons for adjustment of the antibacterial drug programme need to be recorded in detail. Taking conventional treatment once right heart failure or heart rhythm disorders happened. In addition, other Chinese or Western medicines for phlegm and cough indications of COPD (except those for patients with COPD long-term basic treatment) should be prohibited.

\section{Outcome measure}

Because overall conditioning is the core values of TCM, there is no single indicator could be able to predict patients' recovery to evaluate effect of XJXBCQ granules. Thus, a comprehensive assessment is required to AECOPD. ${ }^{14}$

Clinical symptoms (cough, phlegm, defecation, dyspnoea) score We will record the colour, ${ }^{15}$ viscosity and amount of sputum ${ }^{16}$ daily during the study. Scoring and grading constipation symptoms according to the severity of constipation score; The severity of dyspnoea will be assessed by using a modified British Medical Research Council Respiratory Questionnaire.

\section{TCM syndrome score}

Refer to the Guidelines for TCM Diagnosis and Treatment of COPD (2011) and Guidelines for Clinical Research of New TCM for Chronic Bronchitis (2002), we conduct comprehensive evaluation on cough, phlegm, dyspnoea, defecation, abdominal distension, fever from perspective of the heat-phlegm and sthenic-fu syndrome. The evaluation criteria of TCM syndrome score are shown in table 3. Efficacy index $(\mathrm{n})=[$ (preintervention scores-postintervention scores)/preintervention scores ] $\times 100 \%$. 
Table 3 Evaluation criteria of TCM syndrome score

\begin{tabular}{|c|c|c|c|c|}
\hline Syndrome & Normal (0) & Mild (3) & Moderate (6) & Severe (9) \\
\hline Cough & Not significant & In the morning only & Occasionally at any time & Frequently \\
\hline Phlegm & Not significant & $\begin{array}{l}10-50 \mathrm{~mL} \text { in } 24 \text { hours or } \\
\text { dilute white sputum }\end{array}$ & $\begin{array}{l}50-100 \mathrm{~mL} \text { in } 24 \text { hours or sticky } \\
\text { yellow sputum easily expectorated }\end{array}$ & $\begin{array}{l}>1000 \mathrm{~mL} \text { in } 24 \text { hours or sticky } \\
\text { yellow sputum difficultly } \\
\text { expectorated }\end{array}$ \\
\hline Dyspnoea & Not significant & $\begin{array}{l}\text { Occasionally but not } \\
\text { impacting sleep or activity }\end{array}$ & $\begin{array}{l}\text { Accompanying with activity but } \\
\text { improved after rest }\end{array}$ & $\begin{array}{l}\text { Too drastic to be supine position } \\
\text { or sleep or do other activities }\end{array}$ \\
\hline $\begin{array}{l}\text { Abdominal } \\
\text { distension }\end{array}$ & Not significant & A little & Occasional & Continuous \\
\hline Fever & Not significant & $37.5^{\circ} \mathrm{C}-38.0^{\circ} \mathrm{C}$ & $38.1^{\circ} \mathrm{C}-39.0^{\circ} \mathrm{C}$ & $>39.0^{\circ} \mathrm{C}$ \\
\hline
\end{tabular}

TCM, traditional Chinese medicine.

Clinical recovery: TCM clinical symptoms and signs disappeared or approximately disappeared, TCM scores decreased $\geq 90 \%$.

Markedly effective: TCM clinical symptoms and signs are significantly improved, syndrome scores reduced $\geq 70 \%$.

Effective: TCM clinical symptoms and signs are improved, syndrome scores reduced $\geq 30 \%$.

Invalid: No TCM clinical symptoms or signs significantly improved and even aggravated, syndrome scores reduced less than $30 \%$.

\section{Blood gas analysis}

We will take arterial blood gas analysis $\left(\mathrm{PH}, \mathrm{PaO}_{2}\right.$ and $\mathrm{PaCO}_{2}$ ) before and after intervention.

\section{Serum inflammatory markers}

Serum inflammatory markers include PCT, CRP, IL-6, TNF- $\alpha$.

\section{Induced sputum and stool sample}

Induced sputum and stool sample will be collected at the baseline and day 6. Due to TCM characteristic theory 'the Lung and the Large Intestine Are Interior-Exteriorl', we also estimate microbial flora in induced sputum and stool sample of participants to explore the pathogenesis of AECOPD at the microbiological level by the $16 \mathrm{~S}$ rRNA gene sequencing ${ }^{17}$ and the whole-genome shotgun sequencing. ${ }^{18}$

\section{Theoretical discharge time}

The theoretical discharge criteria ${ }^{19}$ are: (1) patients be considered who can adapt to family medicine; (2) patients can accept a stable inhalation therapy about long-acting bronchiectasis, $\beta 2$ receptor agonist and/or an anticholinergic drug, with or without inhaled glucocorticoids. A short-acting $\beta 2$ agonist ${ }^{20}$ should be administered less than once per 4 hours; (3) patients should be able to walk indoors if they were not bedridden before; (4) eating and sleep is good and not influenced by dyspnoea; (5) stay at clinical stability for 12-24hours; (6) arterial blood gas analysis should be stable for 12-24hours; (7) patients or family members fully understand the correct use of stable period medicines and (8) follow-up and home care plans have been arranged.

Mortality

All-cause mortality and COPD mortality will be calculated, respectively, for the subjects during the study period.

Actual hospitalisation time

Actual hospitalisation time: Hospitalisation time $=$ discharge date - admission date +1 .

The proportion of patients requiring invasive mechanical ventilation during hospitalisation

The proportion of patients requiring invasive mechanical ventilation during hospitalisation=the number of patients with invasive mechanical ventilation during hospitalisation/the total patients .

Proportion of patients transferred to ICU during hospitalisation Proportion of patients transferred to ICU during hospitalisation=the number of patients transferred to ICU during hospitalisation/the total patients.

Readmission rate within 30 days after discharge

Judgement criteria for readmission: subjects are hospitalised again due to COPD or other respiratory illnesses, excluding hospitalisation for other illnesses or purposes. The follow-up will be finished and not continued if one is judged to be readmitted to the hospital during the follow-up period. Readmission rate=readmitted patients / completed follow-up patients.

\section{Safety assessment}

The physical examination will be performed every day from the baseline to end of study. Blood routine, urine routine, liver function, renal function test and ECG will be performed at baseline and day 6. Any adverse event occurs during the study will be observed and recorded in detail. 


\section{Quality control and data management}

Prior to the study, the protocol had been reviewed and revised several times by clinicians, statistics and methodologists. All staff members of the trial are required to participate in a series of trainings to ensure that the personnel involved fully understand the research protocol and SOPs to guarantee accuracy and completeness of clinical data. Regular monitoring will be conducted by phone and email. All data will first be recorded by the assessor on a paper version of the CRF and then electronically dualinput into the Electronical Data Capture(EDC) system. The monitor will periodically review the completion and compliance of the CRF. In order to maintain the objectivity of the data, we will ensure that observers and statisticians are unaware of it. The entire process will be monitored by an independent quality inspector. Beijing Qihuang Pharmaceutical Clinical Research Center is responsible for data management.

\section{Statistical analysis}

Full analysis set (FAS), per-protocol set (PPS) and safety analysis set (SS) will be employed. FAS means an ideal set of all subjects (including all subjects randomised into the group and receiving at least one treatment) as close as possible to the principle of intentional analysis. Missing values for major variables, if once fail to observe whole data of case, we will carry forward the last observation to the absence of test data, and the amount of subjects in each group to evaluate efficacy at the endpoint will be corresponding to the beginning of trail. PPS refers to all cases that meet the stand protocol, have great compliance, use the trial medicine in the range of $80 \%-120 \%$, complete the electronic CRF regulations, the main variables can be measured, the baseline variables are well preserved and have no significant violation to the protocol. SS refers to all subjects who accept at least one time treatment after randomisation. For the continuous variables, the paired t-test will be used to compare the changes of clinical symptom scores preintervention and postintervention, and the covariance analysis model will be used for comparison between groups. The multiplier method will be used to calculate the quartiles $(25 \%, 50 \%$, $75 \%$ ) of time from enrolment to events happened, and bilateral 95\% CI and the incidence rate at each time point after enrolment will be calculated yet. Kaplan-Meier curves will be plotted using the log-rank test to compare theoretical hospital stay and actual hospital stay. For the two categorical variables, such as the recurrence rate of laboratory indicator, all-cause mortality, the proportion of mechanical ventilation, the proportion of patients transferred to the ICU during study, and the proportion of readmission within 30 days after discharge, we will make comparison between groups and calculate the 95\% CI using a centrally stratified $\mathrm{CMH} \chi^{2}$ test according to the classification, indicator, time point, quantity and percentage. Statistical analysis will be performed by SAS V.9.4 software.

\section{Patient and public involvement}

Neither patients nor their family members were involved in the study design. The results of the studies will be widely distributed in scientific reports as well as academic conferences to benefit policy-makers, clinicians and patients.

\section{Ethics and dissemination}

All volunteers will sign the informed consent, which is consistent with the ethical principles set forth in the Declaration of Helsinki. Treatment expenses for participants during study will be reimbursed. Data usage will follow the rules of hospital's data oversight committee. Biological samples will be handled following the national guideline on biological waste management and disposal. The results will be disseminated in international peerreviewed journals and be presented in academic conferences. The results will also be disseminated to patients by telephone, inquiring on patient's poststudy health status during the follow-up.

\section{DISCUSSION}

Before conducting this experiment, we closely retrieved PubMed, Web of Science, Embase, CNKI (including China doctor/master's theses database and China Proceedings conference full-text database), Wan Fang Data, Vip Journal Integration Platform, Chinese BioMedical database (Sinomed) and found no definite evidence for effect and safety of XJXBCQ in the treatment of AECOPD. Therefore, we decide to conduct a multicentre, randomised, controlled clinical trial to closely study its effectiveness and safety. Due to the lack of evidence on the effectiveness of XJXBCQ, we will apply TCM combined with Western medicine to treat AECOPD to ensure participants' compliance and ethical considerations, because antibiotics, bronchodilators and glucocorticoids are first-line treatments to improve AECOPD according to guidelines.

COPD is a common, preventable disease, influencing millions worldwide. It seriously impairs patients' social activities, daily activities and quality of lives. ${ }^{21}$ The high economic burden of AECOPD to families and societies is growing year by year. The extensive clinical experience of using Chinese medicine in the prevention and treatment of COPD in China shows that TCM preparations are effective. Due to the complexity of the pathogenesis of COPD, the recent use of combination therapy has attracted more and more attention, providing new prospects for TCM, which be considered effective in improving clinical symptoms. TCM possess the advantages of being simple, convenient, efficient, inexpensive and without serious adverse reactions. A systematic review suggested that Chinese medicine conspicuously improved the prognosis of patients with COPD but lack of high-quality research hinder the development of evidencebased recommendations for clinical practice. Therefore, we design this critical clinical trial, which has the following advantages: (1) This study is designed as a randomised, double-blind, placebo-controlled, clinical, critical trial from 
the perspective of evidence-based medicine, that is, considered to be the most definitive research method of treatment evaluation; (2) At the same time, this is a multicentre trial be carried out in four comprehensive third-grade firstclass hospitals across China, which improves the external validity and representativeness of the sample and reduces the risk of selection bias; (3) Because clinical symptoms are often accompanied by a certain degree of subjectivity, we combine the core symptoms (cough phlegm, defecation, dyspnoea) score and TCM syndrome score to ensure scientific objectivity; (4) In order to ensure quality, all staff in the study must complete the training of the SOPs of the research programme before recruitment; (5) As the main phase of the programme will be performed during the hospital stay, practitioners will be able to immediately identify and manage adverse reactions to guarantee the safety of participants. Practitioners will maintain good communication with the participants by phone after discharge. However, the design of the programme also has potential limitations, for example, the 5-day treatment period and the 1-month follow-up period are a bit short.

In conclusion, the aim of this study is to answer whether TCM can supplement and reduce COPD Western medical treatments, reduce frequency of AECOPD, and provide objective data about effectiveness and safety. If the trial is successful, it will provide patients and physicians with a new option of combining XJXBCQ with Western medicine for better disease remission, which can be implemented on a larger scale in clinical and community settings. ${ }^{22}$ As an innovative and potentially cost-effective strategy, this approach can reduce the disease and financial burden of AECOPD. Given the high prevalence of COPD and serious consequences of acute exacerbations in this group of people, the results of this study can be used to provide information on future international guidelines.

\section{Trial status}

Recruitment started in October 2018 and is expected to finish in March 2020, 18 months in total.

\section{Author affiliations}

'Department of Graduate School, Beijing University of Chinese Medicine, Beijing, China

${ }^{2}$ Department of TCM Pulmonary Diseases, Center of Respiratory Medicine, ChinaJapan Friendship Hospital; National Clinical Research Center for Respiratory Diseases, Beijing, China

${ }^{3}$ Department of TCM Pulmonary Diseases, China-Japan Friendship Hospital, Beijing, China

${ }^{4}$ Department of Pulmonary disease, Wangjing Hospital of China Academy of Chinese Medical Sciences, Beijing, China

${ }^{5}$ Hospital Management Office, Tianjin University of Traditional Chinese Medicine, Tianjin, China

${ }^{6}$ Department of Pulmonary disease, Affiliated Hospital of Tianjin University of TCM, Tianjin, China

${ }^{7}$ Department of Innovation and Transformation, National Center for Traditional Chinese Medicine, State Administration of Traditional Chinese Medicine of the People's Republic of China, Beijing, China

${ }^{8}$ Department of Medical Science Services, Beijing Qihuang Medicine Clinical Research Center, Beijing, China

${ }^{9}$ Department of Pulmonary Medicine, Zhongshan Hospital Fudan University, Shanghai, China
${ }^{10}$ Department of Pulmonary disease, Shanghai Jiao Tong University Medical School Affiliated Ruijin Hospital, Shanghai, China

${ }^{11}$ Department of Respiratory and Critical Care Medicine, China Medical University First Hospital, Shenyang, China

Acknowledgements The authors would like to acknowledge Beijing Qihuang Pharmaceutical Clinical Research Center for managing the trail, Anhui Jiren Pharmaceutical for produce and package of granules. Most especially, we would like to thank the patients with AECOPD who participate in this study.

Contributors $\mathrm{JJ}$ and $\mathrm{HZ}$ are cofirst authors of this manuscript, contributing equally to the design, conduct of the trials and drafting the manuscript. All authors participated in the design of the study and performed the trial. HZ, YZ, TC and $\mathrm{XL}$ supervised and coordinated the clinical trial. JJ, DL, YJ, JZ, QC and EL are responsible for recruiting the participants. ZS and JF participated in the statistical design. All authors read and approved the final manuscript.

Funding The study is financed by National Key Research and Development Program of China (2017YFC1309305).

Competing interests None declared.

Patient consent for publication Not required.

Ethics approval This trail has been approved by the Ethics Committee of ChinaJapan Friendship Hospital.

Provenance and peer review Not commissioned; externally peer reviewed.

Open access This is an open access article distributed in accordance with the Creative Commons Attribution Non Commercial (CC BY-NC 4.0) license, which permits others to distribute, remix, adapt, build upon this work non-commercially, and license their derivative works on different terms, provided the original work is properly cited, appropriate credit is given, any changes made indicated, and the use is non-commercial. See: http://creativecommons.org/licenses/by-nc/4.0/.

\section{REFERENCES}

1 GOLD. Global strategy for the diagnosis, management and prevention of COPD. Global Initiative for Chronic Obstructive Lung Disease 2018.

2 Wang C, Xu J, Yang L, et al. Prevalence and risk factors of chronic obstructive pulmonary disease in China (the China Pulmonary Health $[\mathrm{CPH}]$ study): a national cross-sectional study. The Lancet 2018;391:1706-17.

3 Lareau S, Moseson E, Slatore CG. Exacerbation of COPD. Am J Respir Crit Care Med 2018;198:P21-2.

4 Pavord ID, Jones PW, Burgel P-R, et al. Exacerbations of COPD. Int $J$ Chron Obstruct Pulmon Dis 2016;11 Spec Iss:21-30.

5 Liu M, Zhong X, Li Y, et al. Xuan BAl Cheng Qi formula as an adjuvant treatment of acute exacerbation of chronic obstructive pulmonary disease of the syndrome type phlegm-heat obstructing the lungs: a multicenter, randomized, double-blind, placebo-controlled clinical trial. BMC Complement Altern Med 2014;14:239.

6 Cheng T, Gong Y, Guo Y, et al. Systemic corticosteroid for COPD exacerbations, whether the higher dose is better? A meta-analysis of randomized controlled trials. Clin Respir J 2013;7:305-18.

7 Fermont JM, Masconi KL, Jensen MT, et al. Biomarkers and clinical outcomes in COPD: a systematic review and meta-analysis. Thorax 2019;74:439-46.

8 Lozo Vukovac E, Miše K, Gudelj I, et al. Bronchoalveolar pH and inflammatory biomarkers in patients with acute exacerbation of chronic obstructive pulmonary disease. J Int Med Res 2019;47:791-802.

9 Tsay JJ, Segal LN. Can the sputum microbiota be a biomarker that predicts mortality after acute exacerbations of COPD? Am J Respir Crit Care Med 2018.

10 Izquierdo JL, Cosio BG. The dose of inhaled corticosteroids in patients with COPD: when less is better. Int $J$ Chron Obstruct Pulmon Dis 2018;13:3539-47.

11 Celli BR, Barnes PJ. Exacerbations of chronic obstructive pulmonary disease. Eur Respir J 2007;29:1224-38

12 Khateeb J, Fuchs E, Khamaisi M. Diabetes and lung disease: a neglected relationship. The Review of Diabetic Studies 2018;15:1-15.

13 Barjaktarevic I, Kaner R, Buhr R, et al. Bronchodilator responsiveness or reversibility in asthma and COPD - a need for clarity. Int J Chron Obstruct Pulmon Dis 2018;13:3511-3.

14 Oliveira A, Afreixo V, Marques A. Enhancing our understanding of the time course of acute exacerbations of COPD managed on an outpatient basis. Int J Chron Obstruct Pulmon Dis 2018;13:3759-66. 
15 Allegra L, Blasi F, Diano P, et al. Sputum color as a marker of acute bacterial exacerbations of chronic obstructive pulmonary disease. Respir Med 2005;99:742-7.

16 Soler N, Agusti C, Angrill J, et al. Bronchoscopic validation of the significance of sputum purulence in severe exacerbations of chronic obstructive pulmonary disease. Thorax 2007;62:29-35.

17 Woese CR, Fox GE. Phylogenetic structure of the prokaryotic domain: the primary kingdoms. Proc Natl Acad Sci U S A 1977;74:5088-90.

18 Green ED. Strategies for the systematic sequencing of complex genomes. Nat Rev Genet 2001:2:573-83.
19 (AECOPD) EGoDaToAEoCOPD. Chinese expert consensus on the diagnosis and treatment of acute exacerbation of chronic obstructive pulmonary disease (AECOPD). Int $J$ Respir 2017;37:1041-57.

20 Kopsaftis ZA, Sulaiman NS, Mountain OD, et al. Short-Acting bronchodilators for the management of acute exacerbations of chronic obstructive pulmonary disease in the hospital setting: systematic review. Syst Rev 2018;7:213.

21 Casas-Mendez F, Abadías MJ, Yuguero O, et al. Treatment strategies after acute exacerbations of chronic obstructive pulmonary disease: impact on mortality. PLoS One 2018;13:e0208847.

22 Suissa S, Ariel A. Triple therapy trials in COPD: a precision medicine opportunity. Eur Respir J 2018;52. 